\title{
Variability in GPS Sources
}

\author{
D. L. Jauncey ${ }^{1}$, Edward A. King ${ }^{2}$, Hayley E. Bignall ${ }^{1,3, *}$, J. E. J. Lovell ${ }^{1}$, \\ Lucyna Kedziora-Chudczer ${ }^{1}$, A. K. Tzioumis ${ }^{1}$, S. J. Tingay ${ }^{1, \dagger}$, \\ Jean-Pierre Macquart ${ }^{4}$ and P. M. McCulloch ${ }^{5}$ \\ ${ }^{1}$ Australia Telescope National Facility, CSIRO, PO Box 76, Epping, NSW 1710, Australia \\ 2 Earth Observation Centre, CSIRO, GPO Box 3023, Canberra, ACT 2601, Australia \\ ${ }^{3}$ Department of Physics and Mathematical Physics, University of Adelaide, SA 5005, Australia \\ ${ }^{4}$ Kapteyn Astronomical Institute, University of Groningen, The Netherlands \\ ${ }^{5}$ University of Tasmania, Tasmania, Australia
}

Received 2003 March 14, accepted 2003 March 26

\begin{abstract}
Flux density monitoring data at 2.3 and $8.4 \mathrm{GHz}$ is presented for a sample of 33 southern hemisphere GPS sources, drawn from the $2.7 \mathrm{GHz}$ Parkes survey. This monitoring data, together with VLBI monitoring data, shows that a small fraction of these sources, $\sim 10 \%$, vary. Their variability falls into several categories: sources whose spectral classification is, at best, ephemeral on a timescale of years; sources with a stable GPS classification that vary, but retain their classification; and a small number of sources that exhibit interstellar scintillation, but that maintain a mean GPS spectrum. Existing data on GPS sources with higher frequency peaks, $\geq 3 \mathrm{GHz}$, reveals that many such sources vary. However, the majority of these sources possess a GPS spectrum only during outbursts, and hence should perhaps be classified as ephemeral GPS sources. In addition, significant levels of circular polarisation have been found in a number of GPS sources, both amongst the variables and non-variables. Remarkable amongst these is PKS 1519-273, which possesses strong and variable circular polarisation, and which exhibits IDV in all Stokes parameters.
\end{abstract}

Keywords: galaxies: active — quasars: general

\section{Observations}

We present the results of flux density monitoring of a selection of 34 southern candidate gigahertz peaked spectrum (GPS) sources whose 2.3 and $8.4 \mathrm{GHz}$ flux densities were measured during 65 observing sessions spread roughly uniformly over a 30-month period from 1990 through 1993 (King et al. 1992; King 1994; E.A. King et al., in preparation). The sources were selected from the Parkes 2.7 GHz survey (Bolton, Savage, \& Wright 1979, and references therein), and were given a spectral classification based on the flux density measurements available at the time the sample was drawn up, circa 1990. One source, PKS 1830-211, was soon found to vary, quickly losing its GPS classification. However, it was retained in the monitoring program because of its identification as an Einstein ring (Jauncey et al. 1991).

These sources do not form a complete sample, but rather represent the results collected from a variety of surveys. As the source list was drawn from the Parkes $2.7 \mathrm{GHz}$ survey, the majority of sources have spectral peaks below $\sim 3 \mathrm{GHz}$. The sources are listed in Table 1, together with their optical identifications, magnitudes and redshifts where available, and mean flux densities at 2.3 and $8.4 \mathrm{GHz}$. Errors on the individual flux density measurements are $1.8 \%$ and $0.24 \mathrm{Jy}$ at $2.3 \mathrm{GHz}$, and $5.0 \%$ and $0.13 \mathrm{Jy}$ at $8.4 \mathrm{GHz}$, respectively, and the errors on the

\footnotetext{
* Present address: Joint Institute for VLBI in Europe, Dwingeloo, The Netherlands.

$\dagger$ Present address: Swinburne University of Technology, Hawthorn, Australia.
}

overall mean values in Table 1 are $0.5 \%$ and $0.13 \mathrm{Jy}$, and $1.0 \%$ and $0.02 \mathrm{Jy}$ at 2.3 and $8.4 \mathrm{GHz}$ respectively.

\section{Discussion of the Individual Variable Sources}

This intensive flux density program demonstrated the generally low incidence of flux density variability amongst GPS sources. Significant variation was found in only three sources, PKS 1127-145, PKS 1740-517, and PKS 1830-211.

\subsection{PKS 1127-145}

This quasar showed clear variability at both frequencies, with a smooth decrease at a rate of $0.33 \mathrm{Jy}$ per year that persisted throughout the observations. Despite these variations, the source retained its spectral character throughout the measurement period. Variability at 90 and $230 \mathrm{GHz}$ has also been found (Tornikoski et al. 2001), but it appears to retain a GPS classification at high frequencies in the sense that the spectrum seems to remain optically thin above $10 \mathrm{GHz}$. However, its GPS classification is questioned by Tornikoski et al. (2001).

\subsection{PKS 1740-517}

There is some evidence for weak variations at $2.3 \mathrm{GHz}$, but no variations are apparent at $8.4 \mathrm{GHz}$ in this faint galaxy. Our VLBI observations at $2.3 \mathrm{GHz}$ (E.A. King et al., in preparation) reveal a widely spaced, extended double with separation of 52 mas. The substantial peak in the spectrum, together with the compactness of PKS 1740-517, indicates that the GPS classification remains valid. 
Table 1. Sources included in the survey, with definite/likely GPS sources denoted by a $* /$ ? respectively, after the source name. For the optical identifications, G: galaxy, Q: quasar, EF: empty field

\begin{tabular}{|c|c|c|c|c|c|}
\hline PKS Source & ID & mag & $z$ & $S_{2.3}$ & $S_{8.4}$ \\
\hline $0008-421 *$ & $\mathrm{G} ?$ & 22.0 & & 2.92 & 0.66 \\
\hline $0022-423 *$ & G & 20.6 & 0.937 & 3.08 & 1.04 \\
\hline $0023-263 *$ & G & 20.2 & 0.322 & 6.39 & 2.34 \\
\hline 0159-117* & Q & 16.3 & 0.669 & 2.09 & 0.99 \\
\hline 0237-233* & Q & 16.4 & 2.223 & & \\
\hline $0403-132 ?$ & Q & 16.8 & 0.571 & 3.56 & 2.46 \\
\hline 0624-058* & $\mathrm{G}$ ? & 21.0 & & 12.96 & 4.15 \\
\hline $0704-231 *$ & & & & 2.78 & 1.11 \\
\hline $0733-174 ?$ & $\mathrm{EF}$ & & & 2.77 & 1.44 \\
\hline $0741-063 *$ & $\mathrm{EF}$ & & & 5.71 & 1.69 \\
\hline $0823-500 *$ & & & & 5.38 & 1.54 \\
\hline 0834-196* & $\mathrm{G}$ ? & 19.6 & 1.032 & 3.08 & 0.97 \\
\hline $1015-314^{*}$ & $\mathrm{G} ?$ & 21.2 & 1.346 & 2.50 & 0.92 \\
\hline $1127-145^{*}$ & Q & 17.0 & 1.187 & 5.12 & $2.6-3.4$ \\
\hline $1148-001 *$ & Q & 17.1 & 1.980 & & \\
\hline $1151-348^{*}$ & Q & 17.8 & 0.258 & 4.65 & 1.80 \\
\hline $1215-457 *$ & Q & 19.0 & 0.529 & 3.80 & 1.46 \\
\hline $1221-423 *$ & G & 18.0 & 0.171 & 1.98 & 0.78 \\
\hline $1245-197 *$ & $\mathrm{G}$ & 21.6 & 1.275 & 4.01 & 1.65 \\
\hline $1302-492 ?$ & $\mathrm{G}$ & 9.4 & 0.002 & 5.45 & 2.05 \\
\hline $1306-095^{*}$ & $\mathrm{G}$ ? & 20.5 & 0.464 & 3.47 & 1.41 \\
\hline $1320-446 ?$ & $\mathrm{G}$ ? & 21.0 & & & \\
\hline $1323-611 ?$ & & & & 7.16 & 2.19 \\
\hline $1354-174 *$ & Q & & 3.147 & 1.67 & 0.81 \\
\hline $1733-565 ?$ & $\mathrm{G}$ & 17.2 & 0.098 & 5.80 & 1.93 \\
\hline $1740-517 *$ & G & 20.2 & & $4.9-6.3$ & 2.48 \\
\hline $1827-360 *$ & & & & 3.80 & 0.69 \\
\hline $1830-211$ & & & & $8.8-11.6$ & $4.9-10.9$ \\
\hline 1934-638* & $\mathrm{G}$ & 18.9 & 0.183 & 12.39 & 3.05 \\
\hline $2052-474 ?$ & Q & 19.1 & 1.489 & 2.04 & 1.91 \\
\hline $2126-158^{*}$ & Q & 16.6 & 3.266 & 1.14 & 1.06 \\
\hline $2135-209 *$ & G & 20.4 & 0.639 & 2.69 & 0.97 \\
\hline $2149-287 ?$ & $\mathrm{Q}$ ? & 21.4 & & 2.71 & 0.96 \\
\hline $2311-452 *$ & Q & 19.1 & 2.883 & 1.93 & 0.96 \\
\hline
\end{tabular}

\subsection{PKS 1830-211}

This strong, low Galactic latitude, $b_{I I}=-5.7^{\circ}$, source was included in the GPS sample on the basis of its radio spectrum which was constructed from our own observations plus what was available in the literature circa 1990. This showed that the source had a broad, fairly flat spectrum which appeared to peak near $1 \mathrm{GHz}$, sufficient to justify its inclusion in the sample as a candidate GPS source. Our first VLBI observations showed it to possess two separate milliarcsecond-scale cores separated by 1 arcsecond (King et al. 1992).

Early in the monitoring program PKS 1830-211 was identified as an unusually strong Einstein ring/gravitational lens (Jauncey et al. 1991), so the flux density monitoring assumed an added interest. It was the first source in this observing program to exhibit strong variability, with the $8.4 \mathrm{GHz}$ flux density doubling in the course of a year (Jauncey et al. 1992; King 1994; Lovell et al. 1996). This dramatic variability demonstrated the ephemeral nature of this source's GPS classification. The variability was sufficiently large that we were able to determine a time delay between the two compact components, which, in turn, yielded an estimate of $H_{0}$ (Lovell et al. 1998).

Thus, of the three sources found to vary, only two, PKS 1127-145 and PKS 1740-517, retained their GPS classification while varying. The apparent GPS spectrum of PKS 1830-211 was the accidental result of plotting non-coeval data points, and its GPS spectral classification is at best ephemeral. Two other GPS sources, PKS 0823-500 and PKS 1718-649, have also exhibited low level radio variability, and these are discussed below.

\subsection{PKS 0823-500}

This strong source exhibits a 'classical' GPS spectrum, with a well-defined peak close to $2 \mathrm{GHz}$. The source is within 10 degrees of the Galactic plane, and remains optically unidentified to the magnitude limit of the UK Schmidt survey. It showed no significant variability at 2.3 or $8.4 \mathrm{GHz}$ in the original Mt Pleasant monitoring (King 1994). However, our VLBI observations (King 1994) revealed significant changes in the source visibilities at $2.3 \mathrm{GHz}$ on the Tidbinbilla-Hobart baseline. The two epochs of VLBI observations are presented in Figure 1. In October 1988 the source beat to zero, whereas by December 1989 the beat very clearly did not descend to zero. The source is modelled as two roughly equal components with separation 21.5 mas (King 1994). The changes in visibility present in Figure 1 suggest that there is no significant change in separation; rather, one of the two components has varied in flux density.

The Mt Pleasant flux density monitoring showed no significant evidence for variability. However, as PKS $0823-500$ is a secondary flux density calibrator for the Australia Telescope Compact Array (ATCA), considerable archival data is available for this source. J.M. Chapman (2002, private communication) has undertaken a careful analysis of these data, and finds clear, slow, low-level variability at a level of a few per cent, as expected from the changes seen in the VLBI observations.

\subsection{PKS 1718-649}

PKS 1718-649 is another Parkes GPS source with a radio spectrum that is similar to PKS $0823-500$, but which was not included in the Hobart monitoring program. PKS 1718-649, which has been identified as the nearest GPS galaxy (Tingay et al. 1997) at a redshift of 0.014 (Fosbury et al. 1977), also exhibits radio variability (Tingay \& de Kool 2003). It shows significant variations both above and below the spectral turnover frequency. The observed variability is well fitted by both synchrotron selfabsorption and free-free absorption models, so it is not yet possible to distinguish between these two mechanisms.

\subsection{The Remarkable GPS Source PKS 1519-273}

Another remarkable Parkes source which has been given a GPS classification is PKS 1519-273 (de Vries, Barthel, \& O'Dea 1997). This unusual source is identified with 

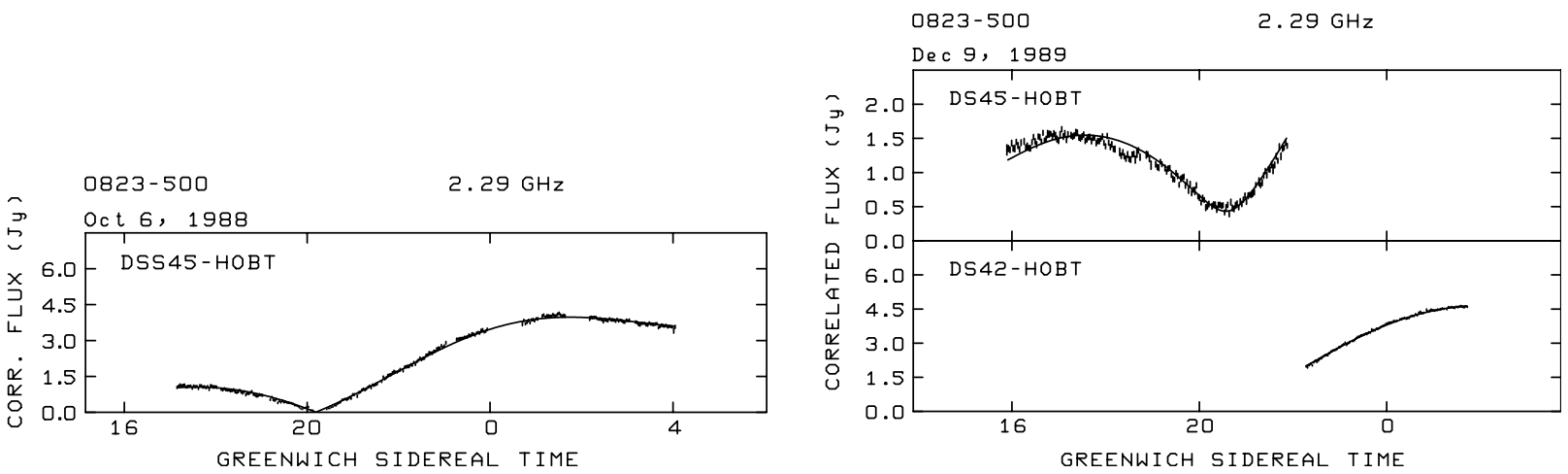

Figure 1 VLBI $2.3 \mathrm{GHz}$ visibility data on the DSS45-Hobart baseline for PKS $0823-500$ from 1988 October and 1989 December. Both data sets show a strong minimum just after $20 \mathrm{~h}$ GST, but the minimum in 1989 December clearly does not beat to zero, as it does in 1988 October.

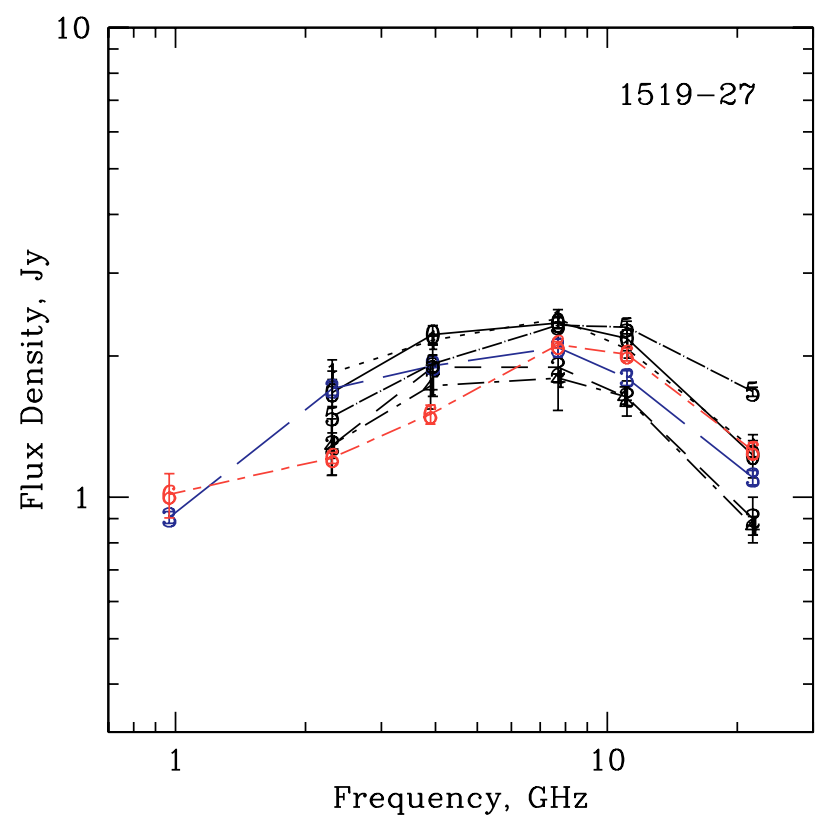

Figure 2 The $\mathrm{cm}$ wavelength radio spectrum of PKS 1519-273 measured with the RATAN 600 radio telescope, from Kovalev et al. (1999).

a 15th magnitude BL Lac object of unknown redshift (White et al. 1988). It is amongst the most compact, submilliarcsecond, radio sources known based on space VLBI observations at $2.3 \mathrm{GHz}$ (Linfield et al. 1989) and $5 \mathrm{GHz}$ (Hirabayashi et al. 2000). The overall spectrum from 0.4 to $22 \mathrm{GHz}$ as determined from measurements at the RATAN 600 over several epochs (Kovalev et al. 1999) is given in Figure 2. It is apparent that, while there is considerable variability, the mean spectrum is clearly GPS with a peak close to $5 \mathrm{GHz}$. SEST flux density measurements confirm that the spectrum remains optically thin to $230 \mathrm{GHz}$ (Tornikoski et al. 2001).

This source was found to exhibit intraday variability (IDV) in the ATCA survey (Kedziora-Chudczer et al. 2001). The source is strongly circularly polarised (Rayner 2000; Rayner, Norris, \& Sault 2000), and detailed multifrequency ATCA observations show a remarkably high fractional amplitude of IDV in all Stokes parameters. We argue that the variability is due to interstellar scintillation (ISS) of a highly compact, 15-35 $\mu$ as, component, possessing $-3.8 \pm 0.4 \%$ circular polarisation at $4.8 \mathrm{GHz}$ (Macquart et al. 2000). A more complete review of the accumulated evidence supporting ISS as the principal mechanism responsible for the IDV seen at $\mathrm{cm}$ wavelengths in many flat spectrum AGN (Heeschen 1984; Witzel et al. 1986; Kedziora-Chudczer et al. 2001) is presented in the proceedings of the NRAO Workshop Radio Astronomy at the Fringe in honour of Ken Kellerman's 65th birthday (Jauncey et al. 2003; Kedziora-Chudczer et al. 2003).

Two basic sets of observations have established the new ISS paradigm. The first is the measurement of significant time delays in the variability patterns of IDV sources between widely spaced radio telescopes of the three fast variables, PKS 0405-385 (Kedziora-Chudczer et al. 1997), J1819+3845 (Dennett-Thorpe \& de Bruyn 2000), and PKS 1257-326 (Bignall et al. 2003). The precision with which the time lag can be determined depends on the characteristic timescale divided by the signal to noise ratio. For these sources this is of order $10-20 \mathrm{~s}$, compared with the time delays of many minutes that have been observed for PKS 0405-385 (Jauncey et al. 2000), J1819+3845 (Dennett-Thorpe \& de Bruyn 2000), and PKS 1257-326 (Bignall 2003; Jauncey et al. 2003). Thus there is little doubt that scintillation is the principal mechanism responsible for the IDV in these sources.

The magnitudes of the time delays observed show that the ISM speeds are $\sim 25 \mathrm{~km} \mathrm{~s}^{-1}$, very close to the $30 \mathrm{~km} \mathrm{~s}^{-1}$ orbital speed of the Earth around the Sun. Thus, for part of the year, when the Earth is moving parallel to the ISM, the relative speed of an Earth-based observer is small and the variability timescale is slow; six months later, the Earth moves in the opposite direction to the ISM, the relative speed is high, and the variability is much more rapid. This gives rise to a clearly visible annual cycle in the variability timescale over the course of a year.

Annual cycles have now been found for three sources, J1819+3845 (Dennett-Thorpe \& de Bruyn 2001), B0917+624 (Rickett et al. 2001; Jauncey \& Macquart 2001), and PKS 1257-326 (Bignall et al. 2003), and there 
is increasing evidence that it is present in a number of other sources exhibiting cm wavelength IDV. This firmly establishes ISS as the principal mechanism responsible for IDV.

For a radio source to scintillate in the ISM its angular size must be of order of, or smaller than, the angular size of the first Fresnel zone. For reasonable distances, tens to hundreds of $\mathrm{pc}$, to the scattering screen, this implies angular sizes of 5 to $30 \mu$ as (Walker 1998) at $\mathrm{cm}$ wavelengths.

Another remarkable property of many of these scintillating sources is the longevity of their scintillation. PKS 1519-273 has exhibited ISS ever since it was discovered in 1993 (Kedziora-Chudczer 1998; Macquart et al. 2000; Kedziora-Chudczer et al. 2001). In this regard it is not alone, as B0917+624 has been scintillating for close to 15 years (Jauncey \& Macquart 2001), although the scintillation recently ceased (Fuhrmann et al. 2002; M.J. Klein 2002, private communication). Archival ATCA data for PKS 1257-326 suggests that it has been scintillating since 1995 (Bignall 2003), and J1819+3845 since its discovery four years ago (Dennett-Thorpe \& de Bruyn 2000). Despite their microarcsecond angular sizes and light-month physical sizes, these scintillating components remain ultra-compact for years or even decades.

\section{Circular Polarisation in GPS Sources}

The discovery of strong, variable circular polarisation in PKS 1519-273 was remarkable, as circular polarisation in extragalactic sources is very small, typically 0.05 to $0.1 \%$ of the total flux density (e.g. Roberts et al. 1975; Weiler \& de Pater 1983). Comparison of the fluctuations in total flux density, I, and in circularly polarised flux density, $\mathrm{V}$, imply that the scintillating component is exceptionally highly circularly polarised at 4.8 and $8.6 \mathrm{GHz}$ (Macquart et al. 2000). Moreover, the high level of correlation between the IDV in I and V demonstrates that the total intensity and circularly polarised components of this source are intimately connected, as the scintillation observations demonstrate that they are coincident and are the same size (Macquart et al. 2000).

A search for radio circular polarisation in a selection of southern AGN has been undertaken with the ATCA (Rayner et al. 2000). The authors note a strong correlation between the presence of circular polarisation and both radio spectral index, in the sense that the more inverted the spectral index, the stronger the circular polarisation, and variability. Since there is a very strong general correlation between variability and spectral index, these findings may reflect the same underlying phenomenon. They also note the low levels of circular polarisation in the non-variable GPS sources in their sample.

Carrying this a step further, Rayner (2000) determined the circular polarisation in a group of 10 Parkes GPS sources, consisting of four GPS galaxies, five GPS quasars, plus the unidentified source PKS 0823-500. Significant circular polarisation was detected in seven of these 10 sources, with the $z=3.27$ GPS quasar PKS 2126-158 showing the strongest circular polarisation of any source yet found. There appeared to be no significant population differences between the quasar and galaxy populations in terms of the presence or absence of circular polarisation, other than the redshift distributions (Rayner 2000), although the sample size is small. However, it is noteworthy that the two sources in Rayner's sample of 41 sources ( 31 from the AGN survey plus 10 GPS sources) that showed the strongest levels of circular polarisation, PKS 2126-158 and PKS 1519-273, are both GPS sources.

\section{Conclusions}

We find that the incidence of flux density variability in GPS sources is generally low, $\sim 10 \%$. A small number of GPS sources show both flux density and structural variations. In our sample, two, PKS 1740-517 and PKS 1127-145, showed significant, though low level, variability, but retained their spectral classification. Two other Parkes GPS sources, PKS 0823-500 and PKS 1718-649, have also shown low-level, though significant, flux density variability, and PKS 0823-500 has also shown some structural variations. There are some sources that may exhibit ephemeral GPS spectral characteristics, but their intrinsic variability is sufficiently strong that they soon pass from this classification. The variable GPS sources most likely represent the slow evolution of the usual month-to-year variability commonly observed in flat spectrum sources, which exhibits GPS characteristics. On a timescale of decades or longer, it may be that more GPS sources will be seen to vary.

In addition, significant levels of circular polarisation have been found in a number of GPS sources, both amongst the variables and non-variables. Remarkable amongst these are the two most strongly circularly polarised sources yet discovered, the BL Lac object PKS 1519-273, which possesses strong and variable circular polarisation, and which exhibits IDV in all Stokes parameters, and the $z=3.27$ quasar PKS 2126-158. The presence of strong ISS in the GPS BL Lac object PKS 1519-273 underlines the heterogeneous nature of the GPS classification.

\section{Acknowledgments}

The ATCA is part of the Australia Telescope, which is funded by the Commonwealth of Australia for operation as a National Facility managed by CSIRO. HEB acknowledges the support of a Faculty of Science Scholarship from the University of Adelaide.

\section{References}

Bignall, H. E. 2003, PhD Thesis, University of Adelaide Bignall, H. E., et al. 2003, ApJ, 585, 653

Bolton, J. G., Savage, A., \& Wright, A. E., 1979, AuJPA, 46, 1

Dennett-Thorpe, J., \& de Bruyn, A. G. 2000, ApJ, 529, L65

Dennett-Thorpe, J., \& de Bruyn, A. G. 2001, Ap\&SS, 278, 101

de Vries, W. H., Barthel, P. D., \& O’Dea, C. P. 1997, A\&A, 321, 105

Fosbury, R. A. E., Mebold, U., Goss, W. M., \& van Woerden, H. 1977, MNRAS, 179, 89 
Fuhrmann, L., et al. 2002, PASA, 19, 64

Heeschen, D. S. 1984, AJ, 89, 1111

Hirabayashi, H., et al. 2000, PASJ, 52, 997

Jauncey, D. L., \& Macquart, J. -P. 2001, A\&A, 370, L9

Jauncey, D. L., Bignall, H. E., Lovell, J. E. J., Kedziora-Chudczer, L., Tzioumis, A. K., Macquart, J. -P., \& Rickett, B. J. 2003, in Radio Astronomy at the Fringe, ASP Conference Series, ed. A. J. Zensus, E. Ros, \& M. H. Cohen (San Francisco: ASP), in press

Jauncey, D. L., \& the SHEVE Team 1992, in Sub-arcsecond Radio Astronomy, ed. R. J. Davis \& R. S. Booth (Cambridge: Cambridge University Press), 134

Jauncey, D. L., Kedziora-Chudczer, L., Lovell, J. E. J., Nicolson, G. D., Perley, R. A., Reynolds, J. E., Tzioumis, A. K., \& Wieringa, M.H. 2000, in Astrophysical Phenomena Revealed by Space VLBI, proceedings of the VSOP Symposium held at the Institute of Space and Astronautical Science, January 2000, ed. H. Hirabayashi, P. G. Edwards, \& D. W. Murphy (Sagamihara, Japan: Institute of Space and Astronautical Science), 147

Jauncey, D. L., et al. 1991, Nature, 352, 132

Kedziora-Chudczer, L. 1998, PhD Thesis, University of Sydney

Kedziora-Chudczer, L., Jauncey, D. L., Wieringa, M. H., Tzioumis, A. K., \& Reynolds, J. E. 2001, MNRAS, 325, 1411

Kedziora-Chudczer, L., Jauncey, D. L., Wieringa, M. H., Walker, M. A., Nicolson, G. D., Reynolds, J. E., \& Tzioumis, A. K. 1997, ApJ, 490, L9

King, E. A. 1994, PhD Thesis, University of Tasmania

King, E. A., \& the SHEVE Team 1992, in Sub-arcsecond Radio Astronomy, ed. R. J. Davis \& R. S. Booth (Cambridge: Cambridge University Press), 152
Kovalev, Y. Y., Nizhelsky, N. A., Kovalev, Y. A., Berlin, G. V., Zhekanis, G. V., Mingaliev, M. G., \& Bogdantsov, A. V. 1999, A\&AS, 139, 545

Linfield, R. P., et al. 1989, ApJ, 336, 1105

Lovell, J. E. J., McCulloch, P. M., King, E. A., \& Jauncey, D. L. 1996, in Astrophysical Applications of Gravitational Lensing, IAU Symposium 172, ed. C. S. Kochanek \& J. N. Hewitt (Dordrecht: Klumer Academic), 347

Lovell, J. E. J., Jauncey, D. L., Reynolds, J. E., Wieringa, M. H., King, E. A., Tzioumis, A. K., McCulloch, P. M., \& Edwards, P. G. 1998, ApJ, 508, L51

Macquart, J.-P., Kedziora-Chudczer, L., Rayner, D. P., \& Jauncey, D. L. 2000, ApJ, 538, 623

Rayner, D. P. 2000, PhD Thesis, University of Tasmania

Rayner, D. P., Norris, R. P., \& Sault, R. J. 2000, MNRAS, 319, 484

Rickett, B. J., Witzel, A., Kraus, A., Krichbaum, T. P., \& Qian, S. J. 2001, ApJ, 550, L11

Roberts, J. A., Cooke, D. J., Murray, J. D., Cooper, B. F. C., Roger, R. S., Ribes, J. -C., \& Biraud, F. 1975, AuJPh, 28, 325

Tingay, S. J., \& de Kool, M. 2003, PASA, in press

Tingay, S. J., Jauncey, D. L., King, E. A., Tzioumis, A. K., Lovell, J. E. J., \& Edwards, P. G. 2003, PASJ, 55, in press

Tingay, S. J., et al. 1997, AJ, 113, 2025

Tornikoski, M., Jussila, I., Johansson, P., Lainela, M., \& Valtaoja, E. 2001, ApJ, 121, 1306

Walker, M. A. 1998, MNRAS, 294, 307

Weiler, K. W., \& de Pater, I. 1983, ApJS, 52, 293

White, G. L., Jauncey, D. L., Savage, A., Wright, A. E., Batty, M. J., Peterson, B. A., \& Gulkis, S. 1988, ApJ, 327, 561

Witzel, A., Heeschen, D. S., Schalinski, C., \& Krichbaum, Th. 1986, MitAG, 65, 239 